\title{
Mass Conserving Models of Vapor Compression Cycles
}

\author{
Christopher R. Laughman Hongtao Qiao \\ Mitsubishi Electric Research Laboratories \{laughman, qiao\}@merl.com
}

\begin{abstract}
Many dynamic models of vapor compression systems experience nonphysical variations in the total refrigerant mass contained in the system when common modeling approaches are used. Rather than use the traditional state variables of pressure and specific enthalpy, the use of density as a state variable can eliminate these variations. A set of test models is developed in Modelica to study the effect of the state variable selection on the overall system charge, and results indicate that this alternative approach has significant benefits for maintaining a specified mass of refrigerant in the cycle.
\end{abstract}

Keywords: vapor compression cycle, simulation, mass conservation

\section{Introduction}

Trends toward increased integration in building and transportation systems, as well as perennial demands for improved system performance, have continued to encourage interest in the development of dynamic models of vapor compression cycles. Such dynamic cycle models can be used for a variety of purposes, including system design, specification, control, and fault diagnostics, and can be applied to a wide variety of residential, commercial and industrial applications to understand and predict the behavior of field-installed systems. These dynamic models can also be coupled with other systems to examine and design the behavior of systems-of-systems to achieve specified requirements for the overall system and satisfy constraints that must be enforced on the physical hardware.

This wealth of interest in dynamic models of vapor compression cycles has resulted in a corresponding growth in both the literature and the number of documented models for these cycles ( $\mathrm{Li}$ et al., 2014b). The Modelica language is particularly appropriate for the development of these system models, due to its objectoriented, declarative, and acausal modeling approach. This can be seen in the variety of references that have been published over the past 15 years regarding models of vapor compression cycles, such as those found in $\mathrm{Li}$ et al. (2014a), among many others.
The performance of physical system models is often evaluated by comparing particular characteristics or outputs of their simulations to the related characteristics of an experimentally observed system. Since, as George E.P. Box said, "all models are wrong, but some are useful," (Box and Draper, 1987), model creators and users must examine the most salient characteristics of their model to ensure that it accurately describes the behavior of interest. This is particularly important for such complex physical systems as vapor compression cycles; it is essential that engineers compare and validate dynamic cycle models against known experimental behavior and data before expecting to obtain reliable model output. One such variable that can easily be compared between simulation and experiment is the the cycle's refrigerant mass inventory, or charge, which is usually known to a fairly high degree of precision, and is also constant over extended time intervals. Such stability and ease of measurement is theoretically well-suited to use in model parameterization and calibration, and is convenient for study in dynamic system models.

Unfortunately, many model formulations for vapor compression cycles demonstrate significant variations in the total system charge (Cecchinato and Mancini, 2012) that do not correspond to observed behavior in experimental systems. This is significant for a few reasons; perhaps the most important of these is that the dynamics associated with the variations in the cycle charge will be coupled to the other system dynamics and introduce aberrant behavior that would not be observed in an experimental system. In addition, the dynamics of the refrigerant mass may also be important of themselves, particularly as pertains to ongoing efforts to develop cycles with minimized refrigerant charge (Corberan et al., 2011). Finally, the relative ease and precision with which the refrigerant mass can be measured, particularly in relation to other quantities such as the specific enthalpy, can be invaluable in calibrating dynamic models of these systems to experimental data.

One contribution to the related field of evaporator charge management was made by Cecchinato and Mancini (2012), in which the authors develop a movingboundary formulation of a single evaporator that conserves refrigerant mass. Previous work related to the dynamics associated with the cycle charge also includes that of Bonilla et al. (2012), in which the authors study 
the effect of system oscillations and numerical instability resulting from variations in the density in an evaporator. Other work with a similar focus includes that of Tummescheit (2002), which discusses both chattering (oscillations around a phase boundary) and the selection of different state variables due to different parameterizations for the equations of state for various fluids.

There are two primary objectives of this paper: exploring the causes of the variations in the cycle charge, and developing an alternative modeling approach that conserves refrigerant mass. This study will be done via the use of a simplified cycle model, developed in Modelica, that eliminates extraneous complexity yet maintains the salient characteristics of models that cause variations in the cycle charge. While common cycle models have many important additional characteristics, such as the use of detailed heat transfer or frictional pressure drop correlations, these characteristics are not essential to the analysis of, or solution to, the variations in the cycle charge. One additional effect that is significant for experimental systems but has been neglected for this initial study is that of the refrigerant oil; while some of the refrigerant charge in experimental systems is inevitably dissolved in the oil and a charge inventory that ignores this effect will inevitably be lower than experimentally observed system charge, the challenges inherent in modeling the refrigerant-oil interactions and the need for initial work in this area elicited a focus on single-component working fluids.

Following this introduction, Section 2 discusses the causes of the variation in the cycle charge in the context of the finite volume pipe model, as well as a method of eliminating these variations. Section 3 presents a discussion of the construction and implementation of the component models used in the simplified cycle models which are both conservative and nonconservative, as well as an approach for initializing these models to achieve a specified cycle charge. The results of simulating these modified models to eliminate the fluctuations in cycle charge are discussed in Section 4, while the final section summarizes the work presented in the paper and suggests fertile areas for exploration future work.

\section{Cycle Mass Variation}

Basic vapor compression cycles consist of a compressor, an expansion valve, and two heat exchangers. Common simulation architectures are designed to take advantage of the different timescales for the dynamics of the different components; since the time constants of the compressor and expansion valve are such smaller than those of the heat exchangers, algebraic models are used for these components, and dynamic models are used for the heat exchangers. One common type of models for the heat exchanger dynamics used in this research are so-called finite volume models, which use the method of lines to discretize the partial differential equations (PDEs) describing the mass, momentum, and energy conservation in the system. The resulting model formulation consists of a set of ordinary differential equations (ODEs) that can be integrated forward in time to study the dynamics of the system, as well as a set of algebraic constraints including those introduced by the compressor and expansion valve models. While the high complexity of the finite volume models makes them somewhat slower than other heat exchanger modeling approaches, their ability to describe spatial variations in the heat exchanger behavior has made them quite popular (Elmqvist et al., 2003; Franke et al., 2009; Laughman, 2014).

As is the case with the development of any physical system model, it is essential to clearly define the purpose for which a model is constructed to ensure that it uses an appropriate set of assumptions to describe the desired behavior. Since the behavior of the refrigerant mass in the cycle are the focus of this research, the models constructed in this paper only describe the behavior of the working fluid in the pipe, rather than the dynamics of the coupled primary fluid / tube wall / secondary fluid system of a prototypical air-source vapor compression cycle. The conservation equations were also simplified by neglecting both gravitational forces and axial heat conduction in the direction of the fluid flow. Other model assumptions used in this work include that of one-dimensional pipe flow, thermodynamic equilibrium in each discrete volume of the refrigerant pipe at each instant in time, and a homogeneous flow field in the two-phase region, meaning that the liquid and vapor velocities are equal. These assumptions were employed to avoid additional complexity in the models in an effort to focus on the underlying causes of variations in the cycle mass.

Under these assumptions, the PDEs describing the conservation equations for a volume of fluid in the refrigerant pipe are

$$
\begin{aligned}
& \frac{\partial(\rho A)}{\partial t}+\frac{\partial(\rho A v)}{\partial x}=0 \\
& \frac{\partial(\rho v A)}{\partial t}+\frac{\partial\left(\rho v^{2} A\right)}{\partial x}=-A \frac{\partial P}{\partial x}-F_{f} \\
& \frac{\partial(\rho u A)}{\partial t}+\frac{\partial(\rho v h A)}{\partial x}=v A \frac{\partial P}{\partial x}+v F_{f}+\frac{\partial Q}{\partial x},
\end{aligned}
$$

where additional information about the symbols and nomenclature used in these equations can be found in the table at the end of this paper. The Reynolds transport theorem can be used to relate the changes in state for a control volume of fixed dimension to the the fluid flowing into and out of that control volume. The resulting expressions can then be discretized to generate a set of 
ODEs, e.g.,

$$
\begin{aligned}
\frac{d\left(\rho_{j} V_{j}\right)}{d t}= & \dot{m}_{k}-\dot{m}_{k+1} \\
\frac{d\left(\dot{m}_{i} l\right)}{d t}= & \rho_{j} v_{j}^{2} A_{j}-\rho_{j+1} v_{j+1}^{2} A_{j+1}+ \\
& \frac{A_{j}+A_{j+1}}{2}\left(P_{j+1}-P_{j}\right)+F_{f, i} \\
\frac{d\left(\rho_{j} u_{j} A_{j}\right)}{d t}= & \dot{H}_{k}-\dot{H}_{k+1}+ \\
& v_{j} A_{j}\left(P_{j+1}-P_{j}\right)+v F_{f, i}+\dot{Q}_{j},
\end{aligned}
$$

where the set of ODEs corresponds to the number of volumes used to subdivide the length of the refrigerant pipe, and the indices refer the fact that we are using a staggered flow grid (Patankar, 1980). In these equations, the $i$ indices are referred to the momentum grid, the $j$ indices are referred to the thermal grid, and the $k=j+1$ indices refer to the boundaries of the thermal grid. In addition, the term $\dot{H}_{k}$ is defined as

$$
\dot{H}_{k}=\dot{m}_{k} \bar{h}_{\text {upstream }, j}
$$

and the mixed-cup specific enthalpy $\bar{h}$ is equal to the in situ specific enthalpy under the homogeneous flow assumption (Laughman, 2014).

Thermodynamic property relations also play an important role which is complementary to the differential equations of fluid motion. These property relations, which are also algebraic, describe the relations between the intensive and extensive fluid properties for a given volume of fluid in thermodynamic equilibrium. These properties include temperature, pressure, specific enthalpy, and density, among many others. As a result of the Gibbs phase rule, there are two degrees of freedom for a single-component pure fluid when there is only one phase present, so that knowledge of two intensive properties is sufficient to determine any other property. When there are two-phase flows, there is only one degree of freedom, but the specification of an intensive mixture property is also needed to determine the state of the twophase mixture (Bejan, 2006). For example, the specification of pressure $P$ and mixture specific enthalpy $h$ will theoretically allow the calculation of any other properties in the thermodynamic phase space.

The calculation of thermophysical properties for dynamic simulation generally needs to be very fast and accurate, due to the number of function evaluations used in a typical system model. As a result, the use of standard equations of state is discouraged in favor of other interpolating methods, such as cubic polynomials or splines (Aute and Radermacher, 2014). Such methods use function approximation to describe each of a set of desired properties as a function of a much more limited set of properties that are calculated at each time step in the simulation. Many thermophysical property routines for refrigerants use $P$ and $h$ as coordinates in the function approximation space to quickly calculate the variety of necessary properties.

The construction of a dynamic model of a refrigerant pipe must take into consideration both the structure of the equations of fluid motion, as well as the implementation of the thermophysical property calculation methods, to generate a computationally efficient simulation. The selection of an infelicitous set of coordinates in which to integrate the conservation equations 4-6 can result in the generation of a large set of nonlinear equations that must be solved to calculate the fluid properties at every time step and for every fluid volume, resulting in potential numerical and practical challenges.

The most common approach taken in this regard is the selection of pressure $P$ and specific enthalpy $h$ as the state variables for the equations of motion, since these are often also used as the coordinates for calculating the fluid properties. The derivatives of $M(P, h)$ and $U(P, h)$ in the above equations can thus be written as

$$
\begin{aligned}
\frac{d M}{d t} & =V\left(\frac{d \rho(P, h)}{d t}\right) \\
& =V\left(\left.\frac{\partial \rho}{\partial P}\right|_{h} \frac{d P}{d t}+\left.\frac{\partial \rho}{\partial h}\right|_{p} \frac{d h}{d t}\right) \\
\frac{d U}{d t} & =V\left(\frac{d(\rho(P, h) u(P, h))}{d t}\right) \\
& =V\left[\left(\left.h \frac{\partial \rho}{\partial P}\right|_{h}-1\right) \frac{d P}{d t}+\left(\left.\frac{\partial \rho}{\partial h}\right|_{p} h+\rho\right) \frac{d h}{d t}\right] .
\end{aligned}
$$

The use of these property relations, along with the stateSelect attribute, can help the Modelica compiler to select $P$ and $h$ as the state variables for the model. By selecting these properties as state variables, they can be integrated by the solver used in a given Modelica tool, such as DASSL or Radau IIa.

The selection of a set of coordinates for the system can have a significant impact on many other variables of the system. One particular variable that is strongly affected by this choice of state variables is the total mass of the system $M_{\text {total }}$. Since no mass is stored in the compressor or expansion valve models, an expression for $M_{\text {total }}$ can be developed by summing all of the masses for the individual control volumes in the pipe model, e.g.,

$$
M_{\text {total }}=\sum_{k} \rho_{k} V_{k}=\sum_{k} \rho_{k}(P, h) V_{k} .
$$

Because the integration of the state variables results in some error, however, it is important to note that a more accurate description of this sum might be

$$
M_{\text {total }}=\sum_{k} \hat{\rho}_{k}(P+\varepsilon, h+\varepsilon) V_{k},
$$

where $\varepsilon$ is the error tolerance of the integration routine and $\hat{\rho}$ represents the numerical approximation of 
$\rho$. While these integration errors are not problematic in many fluids for which the relation between $P, h$, and $\rho$ is nearly linear, two-phase refrigerant flows experience large changes in density as the fluid passes from the liquid region into the two-phase region. These large density derivatives can effectively amplify small deviations in either $P$ or $h$, resulting in large changes in the density between subsequent time steps. Consequently, small errors in the integration of both of these quantities can accumulate quickly and lead to significant and unexpected changes in the total system mass.

Further consideration of Equation 12 suggests an alternative choice of state variables that can reduce these undesirable changes in the refrigerant mass; since the ultimate objective of reducing nonphysical variations in the system charge is equivalent to reducing the errors in the cell density calculations, the selection of $\rho$ as a state variable will allow the integrator to minimize the errors in the density directly, rather than through $\rho(P, h)$. While this choice may appear to be unconventional because of the potential for numerical chattering caused by the large density changes that accompany the movement of the fluid state across the saturated liquid line, the choice of $P$ and $\rho$ as state variables will eliminate the amplification of errors in the density calculation, resulting in a corresponding reduction in the variation of the total system mass.

The alternative formulation of the state variables results in the following expressions for the derivatives of $M(P, \rho)$ and $U(P, \rho)$ for each control volume, e.g.,

$$
\begin{aligned}
\frac{d M}{d t} & =V \frac{d \rho}{d t} \\
\frac{d U}{d t} & =\frac{d(\rho u(P, \rho) V)}{d t} \\
& =V\left[\left(\left.\rho \frac{\partial h}{\partial P}\right|_{\rho}-1\right) \frac{d P}{d t}+\left(\left.\rho \frac{\partial h}{\partial \rho}\right|_{P}+h\right) \frac{d \rho}{d t}\right] .
\end{aligned}
$$

As might be expected, the selection of $\rho$ does also impose additional costs to the simulation. Perhaps the most significant of these is that the use of $\rho$ as a state variable will result in smaller time steps because of the large values of the derivatives at low static qualities of the flow. In addition, the selection of these state variables will also have an effect on the final set of equations that are generated because the change in coordinates will result in the construction of a different set of equations to calculate the remaining fluid properties, such as the calculation of $h(P, \rho)$. In the case that these equations are nonlinear, the simulation time could also be longer than would be for the case with the selection of the original state variables. However, these costs may be outweighed by the benefit of having a constant cycle charge.

Another alternative method for describing the dynamics of the differential control volume involves expanding the number of state variables to include pressure, specific enthalpy, and density. While this approach does result in a larger number of state variables, it has the advantage of simultaneously minimizing the variations in system charge while enabling the use of $P$ and $h$ for calculating other refrigerant properties. Such a method uses the same differential equations as the $(P, \rho)$ model, but also includes the additional ODE

$$
\frac{d h}{d t}=\left.\frac{\partial h}{\partial P}\right|_{\rho} \frac{d P}{d t}+\left.\frac{\partial h}{\partial \rho}\right|_{P} \frac{d \rho}{d t} .
$$

It is also important to note that the set of property derivatives $\partial h / \partial P$ and $\partial h / \partial \rho$ from Equations 14 and 16 do not need to be separately calculated in the property routine to use $P$ and $\rho$ as state variables. The original set of property derivatives can instead be manipulated to provide the needed derivatives, i.e.,

$$
\begin{aligned}
& \left.\frac{\partial h}{\partial P}\right|_{\rho}=-\left.\left.\frac{\partial \rho}{\partial P}\right|_{h} \frac{\partial h}{\partial \rho}\right|_{P} \\
& \left.\frac{\partial h}{\partial \rho}\right|_{P}=\frac{1}{\left.\frac{\partial \rho}{\partial h}\right|_{P}}
\end{aligned}
$$

\section{Mass Conserving Models}

A simplified cycle model, described in the following section, was developed to evaluate the efficacy of these different approaches at maintaining a specified cycle charge. Details about the components and construction of this test cycle model model will be discussed in this section, as well as the means of initializing this cycle to achieve a specified system charge.

\subsection{Component Models}

The simplified cycle model developed in this section includes three components: a refrigerant pipe, a pump, and an "enthalpy adjuster". These components were used to create a system cycle model which maintained mass and energy balances. While the main focus of this work is the refrigerant pipe, the pump is needed to define a relation between the mass flow rate and the pressure drop, so that these variables can be controlled and varied to examine their effect on the total cycle mass. An additional component, referred to as an enthalpy adjuster, was also used to enforce the conservation of energy throughout the system; this component included no pressure drop, but only modified the enthalpy of the working fluid flowing through it so that energy was conserved over the cycle. Neither the pump nor the enthalpy adjuster stored any refrigerant mass; consequently, these components had no state and imposed only algebraic constraints on the system to achieve a desired system balance point. The state variables were therefore only associated with the refrigerant pipe. 
A simplified pipe model, governed by the equations described in Section 2, was developed to test the impact of the state variable selection on the dynamics of the cycle charge. In addition to the governing ODEs, these models also required the inclusion of a set of closure relations describing the heat transfer and the frictional pressure drop. An ideal heat transfer connection was assumed for the sake of simplicity, so that the thermal energy was directly added to the refrigerant stream in each control volume, rather than being governed by the temperature gradients between the refrigerant pipe wall and the bulk fluid. A simplified momentum equation that only accounts for the steady-state frictional pressure drop in both the single and two-phase regions was also assumed, in which

$$
\Delta P=K \frac{\Delta P_{0}}{\dot{m}_{0}^{2}} \dot{m}^{2},
$$

and the nominal values of $\Delta P_{0}, \dot{m}_{0}$, and the adjustable constant of proportionality $K$ were set at the top level of the model. A numerical regularization method was also implemented to improve the numerical robustness of the model for small values of the mass flow rate and pressure drop (Casella et al., 2006).

One feature of the pipe model that was particularly important to this work was the ability to use different models for the relations between the property differentials. This was achieved by implementing the set of differential models as a replaceable model inside the larger pipe model. Each pipe model includes its own differential volume model, but computes the same terms dMs and dUs. While each of these underlying differential models implements different relationships between the properties, the instantiating pipe model only needs to equate the differentials of the mass and internal energy to the terms on the right hand side of Equations 4-6. This is demonstrated in the following simplified excerpt from the refrigerant pipe model.

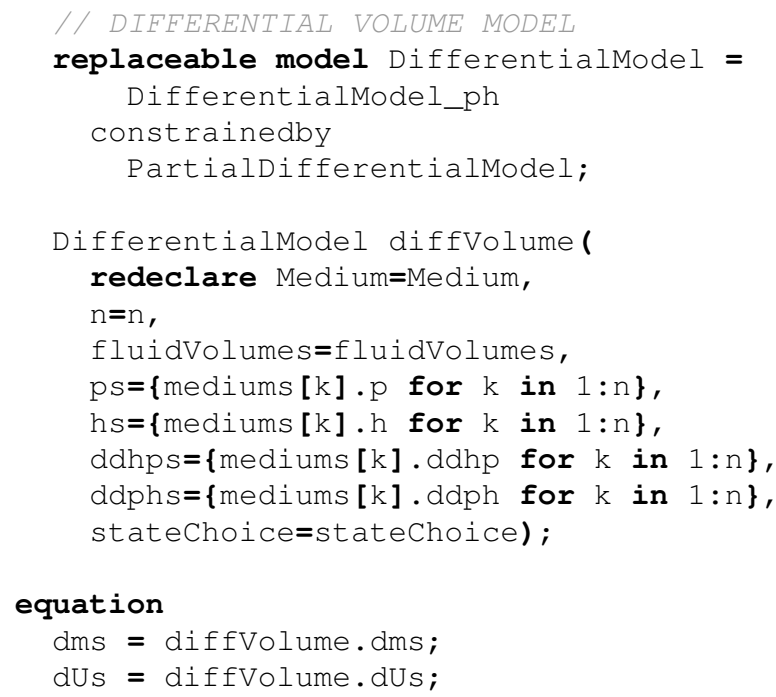

By further establishing a PartialDifferentialModel from which all of these differential models can inherit, the differential volume model can be replaced while maintaining some moderate restrictions on the possible types of replacement, enabling the state variables to be changed without changing any of the other equations in the pipe model.

This implementation of these differential volume models also required the careful use of the stateselect attribute, as the selection of states was based upon the choice of state variables managed with the differential volume model. A Thermostates enumeration with literals including states_ph, states_pd, and states_phd was therefore used to coordinate the use of a given differential volume model and the corresponding state selection attribute for the Modelica compiler.

A pump model and an enthalpy adjuster model were also created to study the closed loop cycle dynamics. The pump model used a scaled version of the basic relationship between mass flow rate and pressure drop (Equation 20) to calculate the pressure rise across the pump for the nominal pump speed that is inversely proportional to the pressure drop for pipe model including a given number of control volumes, e.g.,

$$
\dot{m}=\left(\frac{N}{N_{\text {nom }}}\right) \frac{\dot{m}_{0} \sqrt{\Delta P_{0}}}{\sqrt{\Delta P\left(\frac{N_{\text {nom }}}{N}\right)^{2}}} .
$$

As no mass was stored in this component, the mass flow rates into and out of the pump were equal, and the energy change across the pump was a quadratic function that compensated for the change in enthalpy across the pipe due to pressure loss. This term was much smaller than the energy change in the pipe due to the heat flux into the pipe. As was the case for the pressure drop model of the pipe, regularization methods were also used to compensate for numerical singularities.

An analogous enthalpy adjuster model was also created to compensate for the change in the specific enthalpy across the pipe due to the applied heat flux. This model included no pressure drop or mass storage, and only modified the specific enthalpy for the working fluid travelling to include the effect of the total applied thermal energy gain as the fluid travels through the pipe. Consequently, the equations describing the simplified model used to fulfill the energy balance for the overall system are

$$
\begin{aligned}
\dot{m}_{\text {out }} & =\dot{m}_{\text {in }} \\
P_{\text {out }} & =P_{\text {in }} \\
h_{\text {out }} & =\operatorname{inStream}\left(h_{\text {in }}\right)+\dot{Q}_{\text {in }} / m_{\text {in }} \\
h_{\text {in }} & =\operatorname{inStream}\left(h_{\text {out }}\right)-\dot{Q}_{\text {in }} / m_{\text {in }} .
\end{aligned}
$$

This model is very similar to that of Modelica.Fluid.Pipes.StaticPipe, but also 
includes a change in the outlet enthalpy corresponding to the applied heat gain. Stream connectors and regularization methods around zero mass flow rate were also used in these individual components to improve the numerical robustness of the simulation.

\subsection{Initialization}

The problem of achieving a specified constant charge for a cycle simulation can be effectively split into two related problems: the initialization of the simulation so that the cycle mass starts at the specified value, and the maintenance of the cycle charge at that value over the duration of the simulation. While the previous sections of the paper address how to maintain the cycle charge at a constant value, this brief section addresses the means by which a specific value of the cycle charge may be attained. In general, the total refrigerant mass contained in the cycle at initialization depends on the initial refrigerant state in each volume of the system. Because the refrigerant state at zero mass flow rate is relatively easier to determine, the system was initialized as this condition so that the pump speed was zero and there was no heat flux applied to the pipe or the enthalpy adjuster, and then these inputs were turned on after the conclusion of the initialization transient.

The initial conditions for the system were developed using basic thermodynamic reasoning. The specification of a value of cycle charge $M_{\text {total }}$ for a given system volume $V$ effectively specifies the average density of the fluid in the system $\rho_{\text {init }}$; this specifies one variable that determines the state of the system. Independent specification of one other variable for the system, such as the system pressure $P_{\text {init }}$ at zero pump speed and zero heat flux, determines the state of the refrigerant in the system. The specific enthalpy $h_{\text {init }}$ for every component and control volume can therefore be directly calculated from this refrigerant state in a set of initial equations. Since it is common to initialize most components with pressure and specific enthalpy, these calculated initial values for the pressure and specific enthalpy of the working fluid were then used to initialize all of the components in the system to achieve the desired cycle charge.

\section{Results}

The models described in Section 3 were implemented in Modelica and tested to evaluate the efficacy of the proposed strategy for maintaining a constant cycle mass. Three related models were created with identical geometric parameters and input waveforms. These models used the R410a refrigerant property model included in the AirConditioning/ThermoFluidPro library, written by Modelon (Modelon AB, 2015), as well as the simple relationship between frictional pressure drop and mass flow rate described in Equation 20, where $\Delta P_{0}=500 \mathrm{~Pa}$ and $\dot{m}_{0}=10 \mathrm{~g} / \mathrm{s}$. Other salient parameters of the model are included in Table 1. These models were tested in sim-

Table 1. Common parameters for the test cycle models.

\begin{tabular}{ll}
\hline Parameter Name & Value \\
\hline Pipe diameter & $8 \mathrm{~mm}$ \\
Pipe length & $12 \mathrm{~m}$ \\
Maximum heat input & $130 \mathrm{~W} /$ cell \\
& $(3120 \mathrm{~W}$ total $)$ \\
Initial pressure & $1 \mathrm{MPa}$ \\
Initial system charge & $150 \mathrm{~g}$ \\
Number of pipe control volumes & 24 \\
\hline
\end{tabular}

ulation using Dymola 2015 FD01, and were executed on an i7 PC with $8 \mathrm{G}$ of RAM.

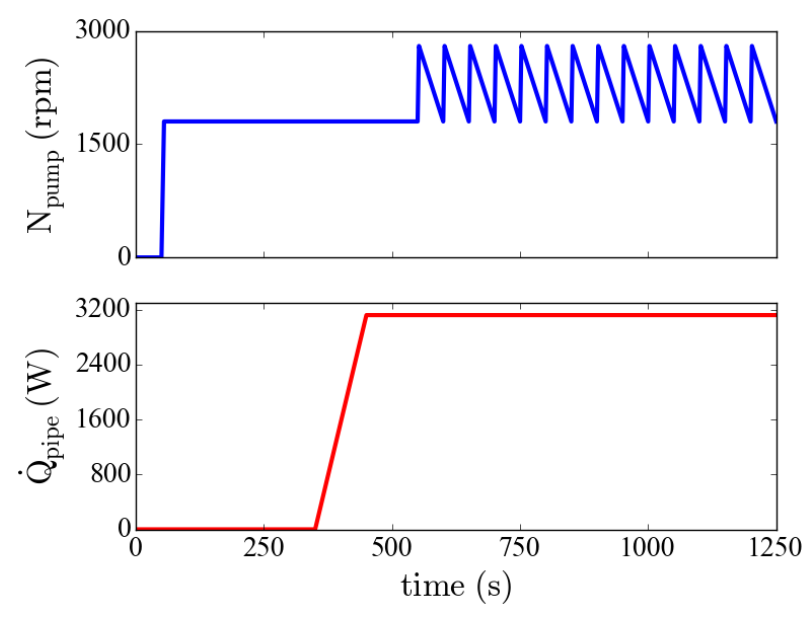

Figure 1. Inputs of pump speed (upper) and heat input (lower) applied to the test cycle.

Because the variations in the cycle charge are related to phase transitions in the fluid volumes across the liquid saturation line, a series of inputs was designed to repeatedly produce these transitions in an effort to induce variations in the cycle charge. These input waveforms, both for the pump speed and the heat source, are illustrated in Figure 1. After the cycle was initialized with the specified refrigerant mass and zero mass flow rate, the pump speed was initially ramped up at 50 seconds from 0 to $1800 \mathrm{rpm}$ over 5 seconds. The resulting transients were then allowed to subside before ramping up the heat source at 350 seconds from 0 to $3120 \mathrm{~W}$ over $100 \mathrm{sec}-$ onds, with the heat being distributed equally over each of the 24 control volumes in the pipe. Finally, a ramp sawtooth waveform was applied to the pump speed to repeatedly cause transitions across the liquid saturation line; the resulting pump speed had a minimum value of $1800 \mathrm{rpm}$, a maximum value of $2800 \mathrm{rpm}$, a period of 50 seconds, and a duty ratio of 0.052 . All of the simulations used identical input waveforms, and were integrated by using the DASSL solver. 


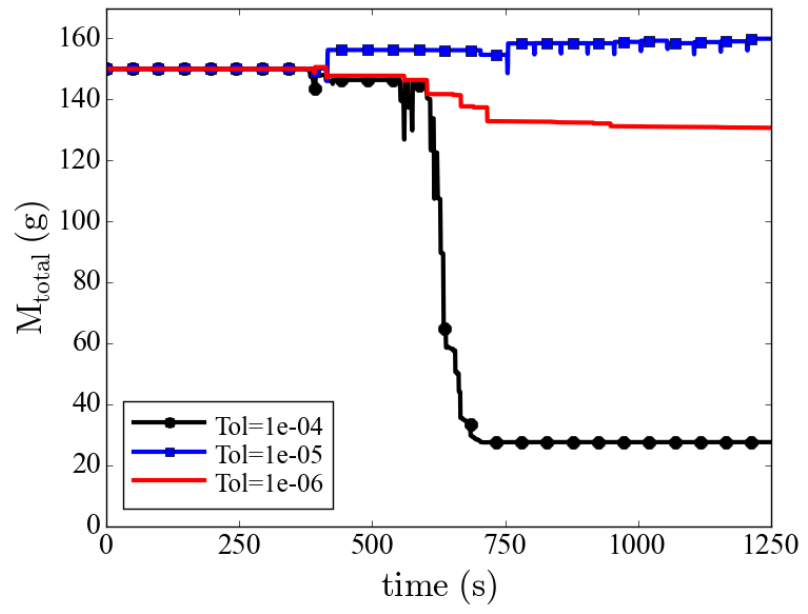

Figure 2. Total cycle mass for three different numerical tolerances with identical applied inputs.

The effect of this waveform on the model using $P$ and $h$ as state variables are illustrated in Figure 2. While many notable features are evident, perhaps the most striking is the amount of variability in the total cycle charge. Such large changes in the total cycle charge can be quite problematic, as they will have a significant impact on the behavior of the cycle. The amount of variation in the total cycle mass is strongly correlated with the tolerance of the solver, suggesting that it is indeed related to the integration tolerances. Moreover, the changes in the mass inventory usually occur by steps, suggesting the presence of a discontinuity that gives rise to these changes.
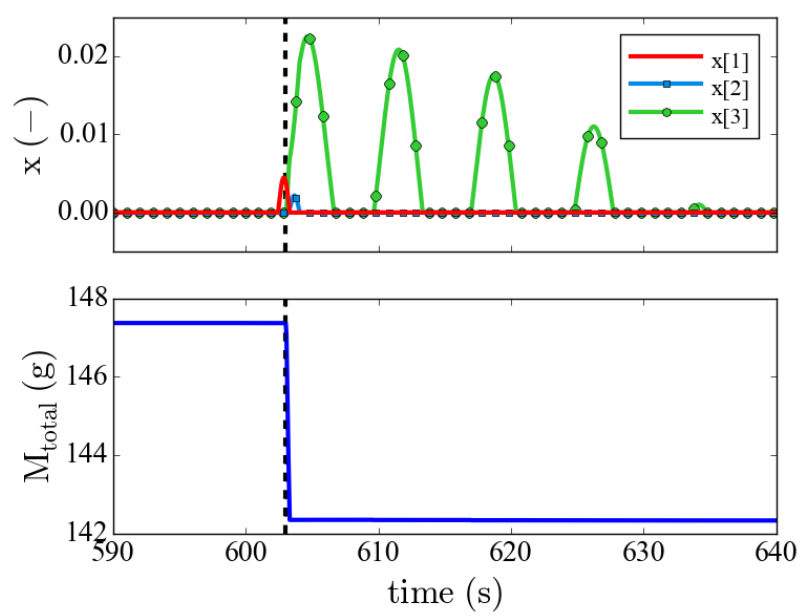

Figure 3. Static quality $x$ at the first, second, and third control volumes in the pipe during the increasing portion of the pump speed waveform, as well as the total system charge at the same moment.

Figure 3 illustrates the relation between the discontinuity caused by the changes in the static quality $x=$ $M_{\text {vap }} / M_{\text {total }}$ for control volumes 1,2, and 3 and the variations in the total system charge. The dashed line drawn at $t=603$ seconds shows a strong correlation between the time that the static quality for all three of these control volumes goes above zero and the time of the step discontinuity in the total system charge. It is also particularly interesting to note that while the quality of the third control volume increases above zero a number of subsequent times in this plot, there are no other variations in the total system charge. This phenomenon suggests that the variations in the refrigerant charge are related not only to a transition across the liquid saturation line, but also to the rate and duration of this transition. The small magnitude of the abrupt excursions over $x=0$ for control volumes 1 and 2 which are associated with large changes in the refrigerant density, as well as the corresponding large changes in the cycle mass, is compatible with the assertion that the variations in the total system charge could be caused by the errors in the state variables.

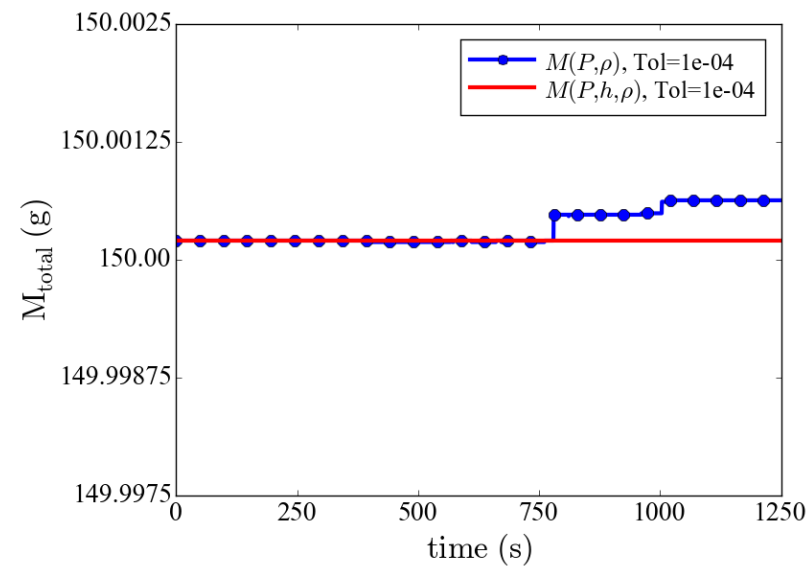

Figure 4. Cycle mass inventory for $M(P, \rho)$ and $M(P, h, \rho)$ models, with an integration tolerance of 1e-04.

In comparison to the large variations in the total system charge exhibited in Figure 2 for the system using $(P, h)$ as state variables, the minuscule variations present in Figure 4 demonstrate that the models that use either $(P, \rho)$ and $(P, h, \rho)$ as state variables have much improved behavior. The variations in the mass for both of these cycles are on the order of 0.25 milligrams, or $1.7 \times 10^{-4} \%$ of the total cycle charge. This compares quite favorably to the output of the simulation of the $(P, h)$ model with the same tolerance, which resulted in an $82 \%$ change in the total cycle charge. Further reductions in the error tolerance for the $(P, \rho)$ and $(P, h, \rho)$ simulations will result in a corresponding reduction in the variation in the total cycle charge.

Additional insights can be gained from the information contained in Table 2, which compares the errors in the simulations and the total time required to run each simulation for different sets of state variables and error tolerances. The errors in this table were generated by calculating the maximum deviation between the total system charge and 150.0 grams, which was the specified charge. As might be expected, the error in the total system charge is far greater for the model with the $(P, h)$ 
Table 2. Max and percentage errors and CPU time for different choices of state variables and integrator tolerances.

\begin{tabular}{lllll}
\hline State Var & Tol & Max Error & $\%$ Error & Time \\
\hline$M(P, h)$ & $1 \mathrm{e}-4$ & $-122.6 \mathrm{~g}$ & $81.7 \%$ & $277 \mathrm{~s}$ \\
& $1 \mathrm{e}-5$ & $3.96 \mathrm{~g}$ & $2.6 \%$ & $127 \mathrm{~s}$ \\
& $1 \mathrm{e}-6$ & $-19.3 \mathrm{~g}$ & $12.8 \%$ & $1925 \mathrm{~s}$ \\
$M(P, \rho)$ & $1 \mathrm{e}-4$ & $1.9 \mathrm{e}-4 \mathrm{~g}$ & $1.2 \mathrm{e}-4 \%$ & $766 \mathrm{~s}$ \\
& $1 \mathrm{e}-5$ & $2.0 \mathrm{e}-4 \mathrm{~g}$ & $1.3 \mathrm{e}-4 \%$ & $1250 \mathrm{~s}$ \\
& $1 \mathrm{e}-6$ & $2.0 \mathrm{e}-4 \mathrm{~g}$ & $1.3 \mathrm{e}-4 \%$ & $1374 \mathrm{~s}$ \\
$M(P, h, \rho)$ & $1 \mathrm{e}-4$ & $2.0 \mathrm{e}-4 \mathrm{~g}$ & $1.3 \mathrm{e}-4 \%$ & $137 \mathrm{~s}$ \\
& $1 \mathrm{e}-5$ & $2.0 \mathrm{e}-4 \mathrm{~g}$ & $1.3 \mathrm{e}-4 \%$ & $315 \mathrm{~s}$ \\
& $1 \mathrm{e}-6$ & $2.0 \mathrm{e}-4 \mathrm{~g}$ & $1.3 \mathrm{e}-4 \%$ & $450 \mathrm{~s}$ \\
\hline
\end{tabular}

state variables than for the other models. One particularly striking and counterintuitive trend is the decrease in the simulation time for the $(P, h)$ models that accompanies the reduction in the tolerance from 1e-4 to 1e-5; this can be attributed to the stiffness of the system of equations during the abrupt changes in the mass inventory in the simulation with the higher tolerance. It is also interesting to note that the simulation time for the $(P, h)$ model with a tolerance of $1 \mathrm{e}-6$ is much greater than for any of the other simulations for any combination of state variables. This can potentially be attributed to the presence of so many discontinuities in the simulation waveform due to the changes in the refrigerant mass; since the solver must take very small time steps past each discontinuity to maintain the specified error tolerance, the sum effect of these discontinuities is that the average time step of the solver must be much smaller than might otherwise be necessary.

Comparison of the simulation time of the $(P, h)$ models to the $(P, \rho)$ models indicates that the $(P, \rho)$ models are slower, as expected, because the large variations in refrigerant density cause the solver to take correspondingly smaller time steps. Finally, it is also evident from Table 2 that the $(P, \rho)$ and $(P, h, \rho)$ methods have identical accuracy for practical intents and purposes, but the time required to run the $(P, h, \rho)$ simulations is much smaller than that of the $(P, \rho)$ simulations. This can potentially be attributed to the nonlinear equations that must be solved to calculate $h(P, \rho)$ when $h$ is not used as a state variable.

\section{Conclusions and Further Work}

Over the course of this paper, the causes of variations in the total system charge were studied and two alternative selections of the state variables that can essentially eliminate such variations were proposed. The effect of these different state variable selections was demonstrated on a simplified cycle model, and the manifestations of the underlying causes for the cycle variation when $P$ and $h$ are solely used as state variables were examined by analyzing the simulation output. While both the $(P, \rho)$ and $(P, h, \rho)$ models had similar accuracy for simulating the total system charge, the $(P, h, \rho)$ models simulated much faster because $h(P, \rho)$ does not have to be calculated when it is also included as a state variable. Moreover, though one ostensible motivation for using $(P, h)$ as state variables is the speed by which the property calculations can be executed, the dynamics associated with the variation in total system charge can somewhat ironically result in simulations that take longer to run than simulations with $(P, \rho)$ as state variables because of the small step sizes required. Models for refrigerant pipes that include either $(P, \rho)$ or $(P, h, \rho)$ as state variables could therefore result in simulations that are both faster and more accurate than might be possible with a choice of $(P, h)$ as state variables.

The results obtained in this work may be extended in a number of directions for future investigation. As suggested in the introduction, an extension of these methods to models which describe the behavior of refrigerant/oil mixtures would be quite valuable. In addition, an error analysis to rigorously demonstrate the causes of these cycle variations would clarify the observations discussed in this paper, and a study of the energy conservation for the system might also provide interesting results. While it is expected that these general trends would hold for different solvers, choices of the nominal attributes of the states, or reference values of the specific enthalpy, further work to explore such trends would be beneficial. Additional study of alternate thermodynamic coordinates might also yield fruitful results; for example, specific entropy is sometimes used to decouple the hydraulic and thermal equations describing fluid flow, and the selection of this or alternate coordinates may also be relevant to these applications. We hope that future studies of these and associated phenomena will continue to yield new insights into these complex and fascinating systems.

\section{Nomenclature}

$\begin{array}{ll}A & \text { cross-sectional area } \\ F_{f} & \text { frictional pressure drop } \\ \dot{H} & \text { enthalpy flow rate } \\ K & \text { proportionality constant for } \dot{m} \rightarrow \Delta P \text { relation } \\ M & \text { mass } \\ N & \text { pump speed } \\ P & \text { pressure } \\ \dot{Q} & \text { heat transfer rate } \\ U & \text { internal energy } \\ V & \text { volume } \\ h & \text { in situ specific enthalpy } \\ \bar{h} & \text { "mixed-cup" specific enthalpy } \\ \dot{m} & \text { mass flow rate } \\ t & \text { time } \\ u & \text { specific internal energy }\end{array}$


$v$ velocity

$\rho$ density

$\hat{\rho}$ numerical approximation of density

\section{References}

V. Aute and R. Radermacher. Standardized polynomials for fast evaluation of refrigerant thermophysical properties. In International Refrigeration and Air-Conditioning Conference at Purdue, 2014.

A. Bejan. Advanced Engineering Thermodynamics. Wiley, 3 edition, 2006.

J. Bonilla, L.J. Yebra, and S. Dormido. Chattering in dynamic mathematical two-phase flow models. Applied Mathematical Modeling, 36:2067-2081, 2012.

G.E.P. Box and N.R. Draper. Empirical Model-Building and Response Surfaces. Wiley, 1987.

F. Casella, M. Otter, K. Proelss, C. Richter, and H. Tummescheit. The Modelica Fluid and Media library for modeling of incompressible and compressible thermo-fluid pipe networks. In Proceedings of the 5th Modelica Conference, 2006.

L. Cecchinato and F. Mancini. An intrinsically mass conservative switched evaporator model adopting the movingboundary method. International Journal of Refrigeration, 35:349-364, 2012.

J.M. Corberan, I. Martinez-Galvan, S. Martinez-Ballester, J. Gonzalvez-Macia, and R. Royo-Pastor. Influence of the source and sink temperatures on the optimal refrigerant charge of a water-to-water heat pump. International Journal of Refrigeration, 34:881-892, 2011.

H. Elmqvist, H. Tummescheit, and M. Otter. Object-oriented modeling of thermo-fluid systems. In Proceedings of the 3rd Modelica Conference, 2003.

R. Franke, F. Casella, M. Sielemann, K. Proelss, M. Otter, and $\mathrm{M}$. Wetter. Standardization of thermo-fluid modeling in Modelica.Fluid. In Proceedings of the 7th Modelica Conference, 2009.

C. Laughman. A comparison of transient heat pump cycle simulations with homogeneous and heterogeneous flow models. In International Refrigeration and Air Conditioning Conference at Purdue University, 2014.

P. Li, Y. Li, J.E. Seem, H. Qiao, X. Li, and J. Winkler. Recent advances in dynamic modeling of HVAC equipment. Part 2: Modelica-based modeling. HVAC\&R Research, 20(1): 150-161, 2014a.

P. Li, H. Qiao, Y. Li, J.E. Seem, J. Winkler, and X. Li. Recent advances in dynamic modeling of HVAC equipment. Part 1: Equipment modeling. HVAC\&R Research, 20(1):136-149, 2014b.

Modelon AB. Air Conditioning Library User Guide, 2015. v1.9.0.
S.V. Patankar. Numerical Heat Transfer and Fluid Flow. Hemisphere Publishing Co., 1980.

H. Tummescheit. Design and Implementation of ObjectOriented Model Libraries using Modelica. PhD thesis, Lund Institute of Technology, 2002. 